\title{
Fallstudie Amag Leasing AG: «Videobasierte Identifikation als Wegbereiter für das Online-Leasing»
}

Esad Ceranic, Patrick Frauchiger, Elke Brucker-Kley, Sandro Graf und Amélie-Charlotte Körner

4.1 Kontext und Ausgangssituation - 52

4.2 Motivation und Zielsetzung - 56

4.3 Umsetzung und Wirkung - 60

4.4 Fazit -65

Literatur - 68 
Die fortschreitende Digitalisierung in der Automobilbranche macht auch vor dem Leasinggeschäft nicht Halt. Neue Technologien bieten Ansatzpunkte Produkte, Prozesse und Geschäftsmodelle zu innovieren. Die vorliegende Fallstudie beschreibt, wie die AMAG Leasing AG die Potenziale der videobasierten Online-Identifikation ausschöpfen will, um Kunden ein durchgängiges Online-Leasing ohne Medienbrüche anbieten zu können. Neben einem bereits durchgängig automatisierten Bewilligungsprozess wird dabei der frühzeitige Einbezug der Kundenperspektive zum Schlüsselfaktor.

\subsection{Kontext und Ausgangssituation}

Die 1980 gegründete AMAG Leasing AG ist seit 2009 Marktführer im Schweizer Fahrzeugleasingmarkt und bewirtschaftet (Stand 2016) rund 130.000 aktive Verträge im Privatkundenund Firmenleasinggeschäft. Sie ist eine zur Careal Holding gehörige Gesellschaft, die für den Schweizer und Liechtensteiner Markt die Finanzierung der Konzernprodukte sicherstellt. Rund $30 \%$ aller in die Schweiz importierten Fahrzeuge stammen aus dem Markenportfolio der AMAG Gruppe, die 2015 einen Umsatz von 4,4 Mrd. erzielte und damit zu den 50 umsatzstärksten Unternehmen der Schweiz zählt. Rund 100 der 5400 Mitarbeitenden der AMAGGruppe sind für die AMAG Leasing AG tätig. AMAG Leasing AG ist eine sogenannte Captive Leasinggesellschaft, das heisst markengebundene Leasinggesellschaft, die die Marken VW, Audi, SEAT, Skoda und VW Nutzfahrzeuge abdeckt.

Für die AMAG Gruppe und so auch für die AMAG Leasing AG stehen die Herausforderungen und Chancen der Digitalisierung in der Automobilindustrie (siehe Infobox) im Zentrum einer Reihe strategischer Initiativen. So wurde für die eigenen Garagenbetriebe eine TabletLösung für die mobile Serviceannahme entwickelt, mit deren Hilfe der Serviceberater mit dem Kunden direkt beim Fahrzeug alle Punkte aufnehmen und Auskunft über Kosten, Dauer etc. geben kann. Mit den Beteiligungen am Zürcher Startup Sharoo und dem stationsungebundenen Catch a Car von Mobility erweitert die AMAG das Geschäftsfeld im Bereich Carsharing und digitale Mobilitätsdienstleistungen. 2016 wurde bei AMAG zum «Jahr der Digitalisierung» erklärt. Mit diesem Fokus wurde der strategische Rahmen für verschiedene Projekte in allen Bereichen der AMAG-Gruppe geschaffen, so auch für die in dieser Fallstudie beschriebenen Aktivitäten zur Weiterentwicklung des Online-Leasings und der dafür eingesetzten videobasierten Online-Identifikation.

\section{Digitalisierung in der Automobilbranche - Reaktionen der Akteure}

Digitalisierung verändert nicht nur Kundenerlebnisse, Geschäftsprozesse und Geschäftsmodelle, sondern hat das Potenzial, ganze Branchen mit ihren Produkten, Dienstleistungen und Wertschöpfungsketten zu transformieren. Dies hat das Beispiel der Musikindustrie bewiesen. In der Automobilbranche scheint genau das aktuell zu passieren und der Wandel birgt grosse Herausforderungen, aber auch Chancen für bestehende und neue Akteure. Treiber sind nicht nur technologische Entwicklungen wie Internet of Things, Elektromobilität oder autonomes Fahren, sondern auch gesellschaftliche und wirtschaftliche Entwicklungen, wie die Überalterung der Gesellschaft, die abnehmende Bedeutung des Autos als Statussymbol oder Ressourcen- und Platzbeschränkungen. Daraus resultierende Trends wie die intermodale Mobilität (die kombinierte Nutzung mehrerer Verkehrsmittel) lassen die Branchengrenzen aufweichen. Sie bringen neue Anbieter ins Spiel, deren Geschäftsmodelle häufig digital geprägt sind (z. B. Uber, Carsharing) und unterstreichen die zunehmend etablierte Einstellung 
«Nutzen statt Besitzen» in einer «Share Economy» (Adam und Meyer 2015). Aber auch traditionelle Akteure, wie Automobilvertrieb und Leasinggeber, die im Mittelpunkt der vorliegenden Fallstudie stehen, sowie Automobilhersteller reagieren auf diesen Wandel der Automobilbranche zur digital geprägten «Mobilitätsindustrie» (Wedeniwski 2015). Berger und Rechenbach (2015) illustrieren diese Reaktionen unter anderem mit folgenden Beispielen:

- Im 2012 eröffneten «Audi City - Showroom der Zukunft» in London stehen nicht mehr als drei physische Fahrzeuge auf $690 \mathrm{~m}^{2}$. Die Beratung basiert auf virtueller Realität und der Verkauf ist von den interaktiven Beratungsflächen räumlich getrennt. Im ersten Halbjahr 2013 wurden $50 \%$ der Autos im Londoner Showroom ohne eine Probefahrt verkauft (Singh 2014).

- Daimler bündelt seit 2014 unter der neuen Dienstleistungsmarke «mercedes me» und dem gleichnamigen Kundenportal alle Onlinedienste und mobile Applikationen rund um das Fahrzeug (connect), dessen Service (assist) und Finanzierung. Auch die KundenCommunity (Soziale Netzwerke) sowie Mobilitätsdienste (z. B. moovel) sind integriert.

- Seit 2013 ist bei der Volkswagen Leasingbörse für Gebraucht- und Jahreswagen in Deutschland die Eintrittspforte für den Kunden nicht mehr der stationäre Handel, sondern die Online-Leasingbörse, die das Geschäft abschliessend an den vom Kunden online gewählten Händler übergibt.

\section{- Status quo: Digitalisierung im Back- und Front-End}

AMAG Leasing stellt sich den Herausforderungen der Digitalisierung bereits seit einigen Jahren und hat die Prozesse und Systeme sowohl im Front- als auch im Back-End verändert und erweitert. Der massivste Eingriff gelang mit der durchgängigen Automatisierung des Leasingbewilligungsprozesses. Die bis dahin von Medienbrüchen und manuellen Arbeiten geprägten Abläufe vom Leasingantrag bis zur Vertragsausstellung wurden durchgängig digitalisiert. Im Rahmen eines strategischen Automatisierungsprojektes war eine Prozessautomatisierungssoftware eingeführt und zahlreiche interne und externe Systeme integriert worden (z. B. CRM, Vertragsmanagement, Zentralstelle für Kreditinformationen/ZEK, CRIF Teledata). Mehr als 200 Geschäftsregeln, die bei der Prüfung und Bewilligung der Leasinganträge zur Anwendung kommen, wurden implementiert. Durch die Anwendung und fortlaufende Optimierung dieser Geschäftsregeln im digitalisierten Prozess kann AMAG Leasing Anträge zunehmend vollständig automatisiert, das heisst ohne manuelle Prüfung, bewilligen. Für bewilligte Anträge können ohne Systembruch die Vertragsunterlagen erstellt werden. Aussichtslose Anträge werden aufgrund hinterlegter KO-Kriterien frühzeitig im Prozess automatisch erkannt und abgelehnt. Die automatisierte Bewilligungsquote, die aktuell bei 20 bis $25 \%$ liegt, wird durch die fortlaufende Optimierung der hinterlegten Geschäftsregeln weiter erhöht (Zielwert: 30 \%). AMAG Leasing konnte damit nicht nur nachweislich die Produktivität steigern (+32\%), sondern schafft Freiraum für komplexe Anträge, die eine vertiefte Abklärung erfordern. AMAG Händler und Kunden profitieren von einem raschen Entscheid in einem durchgängigen Prozess mit deutlich reduzierten Bearbeitungszeiten ( $-70 \%)$, was sich in einer nachweisbar höheren Kundenzufriedenheit niederschlägt. Für AMAG Leasing zeigt sich der durchgängig digitalisierte und standardisierte Prozess nicht nur in einer gesteigerten Effizienz, sondern in hoher Datenqualität, einer optimierten Risikobewertung sowie einer deutlichen Verbesserung der Mitarbeiterzufriedenheit. 
Nicht nur die Arbeitsumgebung für AMAG-Leasing-Mitarbeitende und AMAG-Händler wurde in den letzten Jahren digitalisiert, auch im Front-End wurde investiert. Auf der AMAGWebsite werden mit dem Online-Leasingzertifikat und einem Leasingrechner zwei OnlineTools für leasing-interessierte Kunden angeboten.

Der Online-Leasingrechner erlaubt Kunden - online ohne Eingabe persönlicher Daten, nur mit Auswahl weniger Eckdaten des Wunschfahrzeuges (Marke, Modell, Motorisierung) - die Höhe der monatlichen Leasingrate zu berechnen sowie den Einfluss bestimmter Faktoren wie Laufzeit oder jährliche Laufleistung zu simulieren (• Abb. 4.1).

Das Online-Leasingzertifikat (- Abb. 4.2) ist unabhängig von einem konkreten Fahrzeugwunsch. Leasinginteressierte erhalten nach Eingabe ihrer persönlichen Daten, die auch Angaben zur finanziellen Situation und Lebensunterhaltskosten umfassen, direkt im Anschluss per E-Mail ein sogenanntes Leasingzertifikat, das keine verbindliche Zusage darstellt, aber Auskunft gibt über den finanziellen Rahmen, in dem AMAG ein Leasingangebot unterbreiten kann. Legt der Kunde das Leasingzertifikat beim Händler vor, können über eine Identifikationsnummer die bereits eingegebenen persönlichen Daten für den Leasingantrag abgerufen und verwendet werden. Eine Plausibilisierung und Validierung der online eingegebenen persönlichen Daten findet heute erst beim Händler auf der Grundlage eines amtlichen Ausweis-

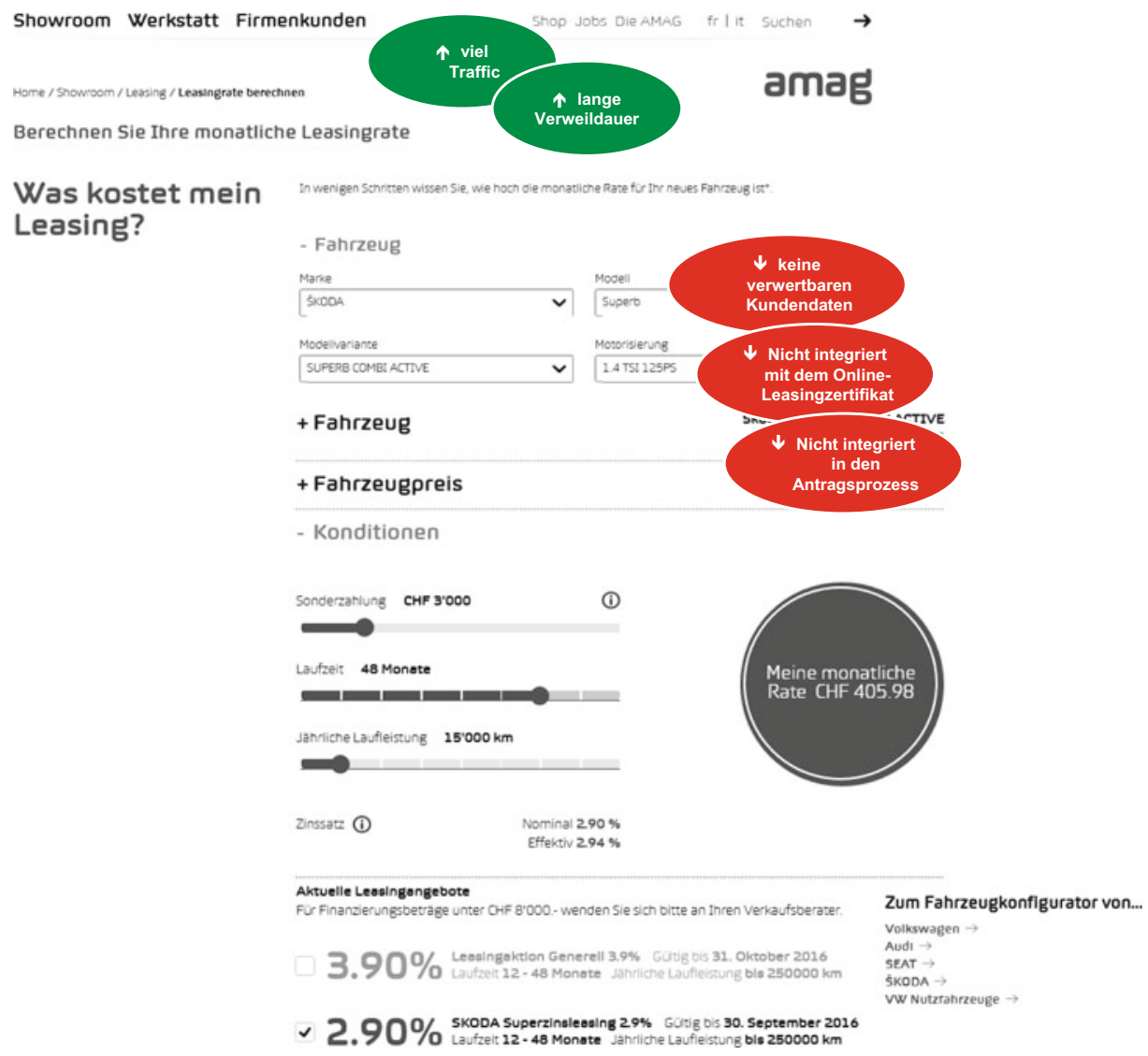

- Abb. 4.1 Online-Leasingrechner. (www.amag.ch, Stand August 2016) 
Home / Snowroom / Leasing / Leasingzertinkat

amag

Leasingzertifikat

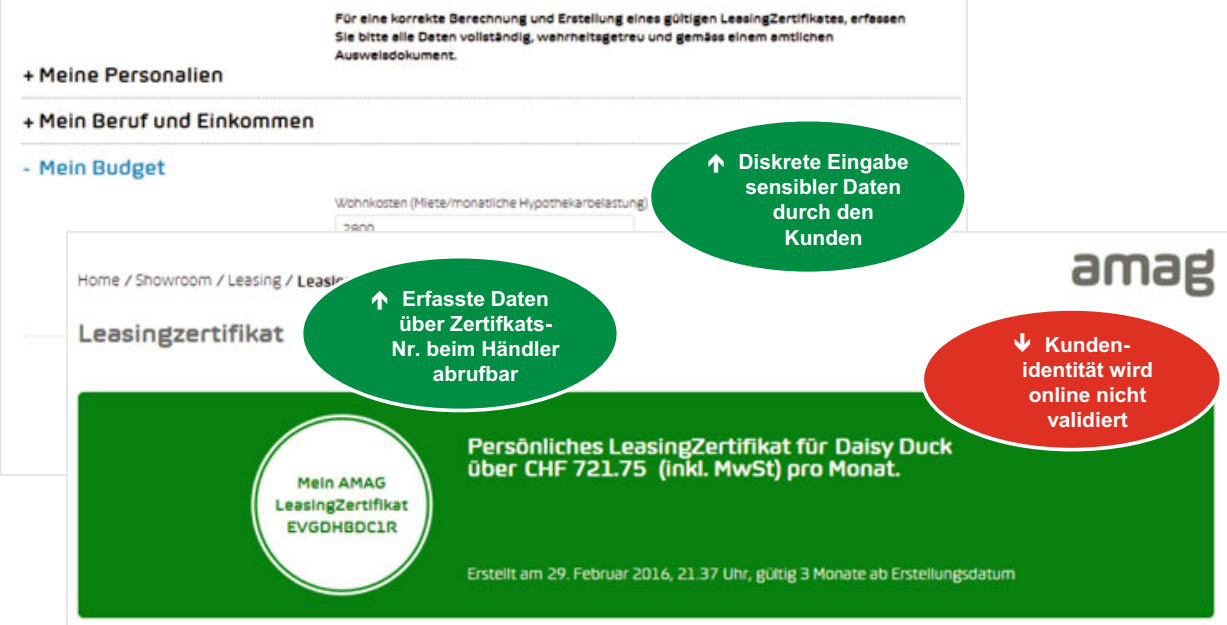

\section{Herzliche Gratulation zu Inrem AMAG LeasingZertifikat}

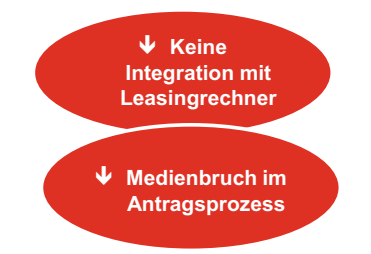

Wir freuen uns, Innen ein Leasing bis zu CHF 721.75 (inkl. MWSt) pro Monat offerieren zu konnen. Mit Ihrem AMAG Leasinezertifikat konnen Sie pei einem offizillen Partner der Marken WW, Audi, SKODA. SEAT, WW Nutzfanrzeuge onne grosse zusatziliche Aufwande und Formalitaten inr Wunschauto aussuchen und Inren Leasingvertrag abschliessen. Ihr personliches Leasingzertifikat basiert aut inren Angaben und den gesetzich vorgeschriebenen Berechnungen, die festiegen, bis zu welchem Betrag Inre monatiche Rate mit den Bestimmungen zur Kreditvergabe in der Schweiz vereinbar ist. *

\section{Fragen zum Leasingzertifikat}

Haben Sie Fragen zu Inrem personlichen Leasingzertfikat oder wünschen Sie eine individuelle Beratung? Von Montag bis Freitag, 8.00 bis 17.30 , sind wir telefonisch unter 0564847650 oder per E-Mail: bestandesmanagement(Bamag, ch fur Sie erreichbar.

- vorbehaitlich Abfrage bei zEKAKO (Zentralstelie fur Kreditinformationen / Informationsstelle fur Konsumkredite)

Als PDF spelchern

$\checkmark$ Wir haben Inr LeasingZertifikat bereits an Ihre E-Mall-Adresse gesendet.

- Abb. 4.2 Online-Leasingzertifikat. (www.amag.ch, Stand August 2016)

dokuments statt. Aktuell sind die beiden Instrumente nicht integriert. Das bedeutet, sie liefern wertvolle Daten, die jedoch nicht in Beziehung zueinander oder zu tatsächlichen Verträgen gesetzt werden können. Der Online-Leasingrechner bietet eine lange Verweildauer (durchschnittlich fünf Minuten) und viel Traffic (12.000 Seitenaufrufe monatlich), aber keine verwertbaren Kundendaten. Somit ist nicht nachvollziehbar, welche und wie viele Leasingverträge aus der Nutzung entstehen. Das Leasingzertifikat hingegen liefert wertvolle Kundendaten, aber durch die fehlende Verifikation der Kundendaten ist nicht eindeutig nachvollziehbar ist, welche Anträge tatsächlich aus einem Online-Leasingzertifikat entstehen. Auch für welche Fahrzeuge sich ein Kunde interessiert, ist nicht ersichtlich. 


\subsection{Motivation und Zielsetzung}

Mit dem vom Antrag, über die Bewilligung bis zur Vertragserstellung und -administration durchgängig digitalisierten Leasingprozess hat AMAG Leasing betriebsseitig die Voraussetzungen geschaffen, um in einem nächsten Schritt den Prozess weiter in Richtung Endkunde zu öffnen. Für Kunden stehen mit dem Online-Leasingrechner und dem Online-Leasingzertifikat bereits zwei Instrumente zur Verfügung, die jedoch nicht in den digitalisierten Leasingprozess eingebunden sind. Die rechtskonforme Feststellung der Kundenidentität spielt hierbei eine Schlüsselrolle. Kann diese in einem digitalisierten Leasingprozess sichergestellt werden, kann AMAG Leasing sowohl den bestehenden Leasingprozess optimieren als auch neue Wege im Online-Leasing einschlagen.

AMAG Leasing unterliegt als Finanzintermediär den Regulatorien zur Bekämpfung der Geldwäscherei und der Terrorismusfinanzierung. Diese umfassen zum einen das Geldwäschereigesetz (GWG 1997) und zum anderen die Verordnung der Eidgenössischen Finanzmarktaufsicht über die Bekämpfung von Geldwäscherei und Terrorismusfinanzierung im Finanzsektor (GwV-FINMA 2015). Darin sind unter anderem die Sorgfaltspflichten bezüglich der Identifikation der Vertragspartei, sprich des Kunden, bei der Aufnahme einer Geschäftsbeziehung festgelegt. Um diesen gerecht zu werden, muss gemäss Art. 45 GwV-FINMA der Kunde persönlich beim Finanzintermediär vorstellig werden, der eine Kopie des Identifizierungsdokuments erstellen, datieren, unterzeichnen und zu den Akten legen muss. Im Fall von AMAG findet diese Identifikation beim Händler statt, der diese Kopien digitalisiert und im Leasingsystem hinterlegt. Wird der Leasingantrag bewilligt und ein Vertrag erstellt, ist AMAG Leasing gemäss GwG verpflichtet, den gesamten Vorgang und somit auch die Identifizierungsdokumente im Rahmen der Vertragsadministration einer Qualitätsprüfung zu unterziehen ( $\bullet$ Abb. 4.3). Bei einem signifikanten Anteil der bearbeiteten Fälle erfüllen die Identifizierungsdokumente die Qualitätskriterien nach GwG/GwV-FINMA nicht (z. B. aufgrund eines schlecht erkennbaren Fotos). In diesen Fällen muss AMAG Leasing den Händler auffordern, den Kunden nochmals in die Garage zu bestellen, um sich erneut zu identifizieren. Das Fahrzeug ist zu diesem Zeitpunkt in der Regel bereits ausgeliefert. Somit schafft diese Prozedur nicht nur unzufriedene Kunden, sondern auch rechtliche Unklarheit, da die Aufnahme der Geschäftsbeziehung bis zur Nachlieferung eines ausreichenden Identifikationsdokuments als abgebrochen gilt (Art. 55 GwV-FINMA). Hinzu kommt ein administrativer Mehraufwand für die Nachbearbeitung, der im Rahmen des Business Cases mit rund 150 Personentagen jährlich quantifiziert wurde.

Ursache der Problematik ist die für alle Akteure umständliche und an den stationären Handel gebundene Form der Identitätsprüfung. Die genannten regulatorischen Vorgaben im Finanzsektor liessen den Einsatz online- und videobasierter Tools für die Identifikation des Vertragspartners bis dato in der Schweiz nicht zu. Die Aufnahme einer Geschäftsbeziehung auf rein digitalem Weg war somit für einen Finanzintermediär nicht möglich. Dies änderte sich jedoch mit dem Erlass des FINMA-Rundschreibens «Video- und Online-Identifizierung» im März 2016, das die Sorgfaltspflichten bei der Aufnahme von Geschäftsbeziehungen über digitale Kanäle regelt (FINMA 2016b). Die FINMA stellt darin die Video- und Online-Identifizierung unter bestimmten Voraussetzungen mit der persönlichen Vorsprache des Kunden gleich (siehe Infobox). 


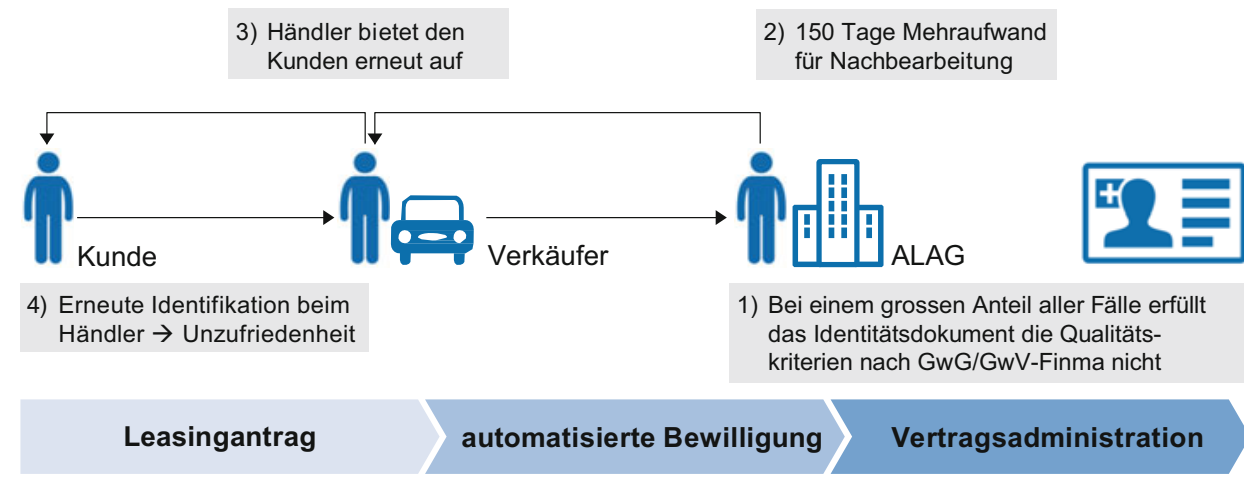

- Abb. 4.3 Auswirkungen nicht GwG/GwV-FINMA-konformer Identitätsdokumente auf den Leasingprozess

\section{FINMA ermöglicht Videoidentifizierung}

Mit Inkrafttreten des FINMA-Rundschreibens «Video- und Online-Identifizierung» im März 2016 (FINMA 2016b), ist es Finanzdienstleistern nun auch in der Schweiz möglich, Kunden bei der Aufnahme einer Geschäftsbeziehung, z. B. bei einer Kontoeröffnung oder einem Leasingantrag, über digitale Kanäle zu identifizieren. Die FINMA stellt damit, wie bereits 2014 die deutsche Aufsichtsbehörde BaFin, die Video- und Online-Identifizierung mit der persönlichen Vorsprache des Kunden gleich. Die FINMA begründet diesen Schritt damit, «unnötige Hürden in der FINMA-Regulierung» abzubauen und die Innovations- und Wettbewerbsfähigkeit des Finanzplatzes Schweiz sicherzustellen (FINMA 2016a). Von den angepassten regulatorischen Rahmenbedingungen profitieren nicht nur Fintech-Startups, sondern alle Finanzdienstleister, die Geschäftsbeziehungen mit ihren Kunden von Beginn an, das heisst bereits beim sogenannten «client onboarding» digital, gestalten wollen.

Ein wesentlicher Grundsatz des Geldwäschereigesetzes und der Verordnung der Eidgenössischen Finanzmarktaufsicht über die Bekämpfung von Geldwäscherei und Terrorismusfinanzierung im Finanzsektor ist die Identifikation der Vertragspartei bei Aufnahme der Geschäftsbeziehung (Art. 3 GwG, Art. 44 GWV-FINMA). Bis zum Inkrafttreten des FINMARundschreibens war die Identifizierung nur durch persönliche Vorsprache mit einem amtlichen Ausweis (inkl. Identitätsfoto) möglich (Art. 45 GWV-FINMA). Als einzige Alternative zur persönlichen Vorsprache war die Zustellung einer echtheitsbestätigten Kopie auf dem Postweg mit einer zusätzlichen Prüfung der Postadresse durch eine Postzustellung vorgesehen. Das FINMA-Rundschreiben erweitert diese Möglichkeiten nun auf digitale Kanäle. So ist neu die Videoidentifizierung von einem beliebigen Endgerät des potenziellen Kunden möglich, sofern die folgenden Grundsätze erfüllt sind (FINMA-RS, Abs. 5-22):

- Die Identifizierung erfolgt mittels audiovisueller Kommunikation in Echtzeit, das heisst in einer Live-Schaltung.

- Die sichere Übertragung und das Auslesen und Entschlüsseln der Information aus der maschinenlesbaren Zone (MRZ) des Ausweises ist sichergestellt.

- Die Bild- und Tonqualität muss eine einwandfreie Identifikation gewährleisten. Während der Videoübertragung müssen Bilder der Vertragspartei und des Identifizierungsdokuments erstellt werden. 
- Alle Angaben nach Art. 44 GwG und Art. 60 GwV-FINMA (Name, Vorname, Geburtsdatum, Adresse, Staatsangehörigkeit) müssen vor der Videositzung erfasst und übermittelt sein. Vor Beginn des Gesprächs muss das ausdrückliche Einverständnis zur Videoidentifizierung eingeholt werden.

- Alle vor und während der Videositzung erhobenen Daten werden abgeglichen, Die Echtheit des Dokuments wird visuell oder mit technischen Mitteln geprüft. Es sind nur Ausweisdokumente zugelassen, die über eine MRZ und optische Sicherheitsmerkmale verfügen.

- Im Laufe der Sitzung ist die Identität der Vertragspartei mittels einer zugestellten Transaktionsnummer (TAN) o. ä. zu verifizieren.

- Die durchführenden Mitarbeitenden müssen geschult und durch einen Gesprächsleitfaden unterstützt sein.

- Der Finanzintermediär kann eine dritte Partei mit der Identifizierung beauftragen. In der Schweiz bieten (zum Zeitpunkt der Studie) Swisscom (»Digital Identification \& Signing «) und die Schweizerische Post («ID-Check») die Videoidentifikation an. Beide Dienste basieren auf der Software der WebID Solutions. Firmenkunden, die diese Anbieter für die Identifikation oder Altersprüfung ihrer Kunden einsetzen, bezahlen pro Vorgang («pay per use»). Die Daten werden an den Dienstleistungsanbieter über eine sichere und verschlüsselte Verbindung übermittelt und anschliessend aus den Systemen des Identifikationsanbieters gelöscht. Auf eine interne Lösung setzt UBS, die im März 2016 eine App für die Videoidentifikation auf der Basis der Softwarelösung IDnow lanciert hat. SowohI WebID als auch IDnow bieten zusätzlich zur rechtssicheren Verifizierung im Video-Chat Funktionen für den digitalen Vertragsabschluss an. Über eine in Echtzeit erstellte elektronische Signatur kann der Kunde im Video-Chat einen Vertrag digital unterzeichnen.

Vor dem Hintergrund dieser aufsichtsrechtlichen Änderung, die bereits im Dezember 2015 von der FINMA angekündigt und in einem Regelungsentwurf zur Stellungnahme kommuniziert wurde, konnte AMAG Leasing rasch reagieren und die Identifikationsproblematik unter neuen Voraussetzungen aufgreifen. Nach Prüfung verschiedener Alternativen entschied sich AMAG Leasing, die Einsatzmöglichkeiten der videobasierten Identifikation für Digitalisierung des Leasings auszunutzen. Das Projekt wurde im Frühjahr 2016 mit folgenden Zielsetzungen gestartet:

- Kurzfristig (Phase 1, Sommer 2016): Optimierung des aktuellen Prozesses durch die Video-Identifikation: Mehraufwände auf Seiten AMAG Leasing, Händler und Kunde, die durch mangelhafte Identitätsnachweise entstehen, werden reduziert.

- Kundennutzen: Der Kunde kann sich bequem im Video-Chat identifizieren. Der erneute Weg zum Händler entfällt.

- Mittelfristig (Phase 2, Herbst 2016): «Online-Bewilligung» (• Abb. 4.4): Das Online-Leasingzertifikat wird durch die Video-Identifikation zur verbindlichen Zusage für einen Leasingvertrag.

- Kundennutzen: Der Kunde kann persönliche Daten über seine finanzielle Situation und Lebenshaltungskosten diskret online erfassen und erhält innert Minuten eine verbindliche Zusage. 
Der Kunde ...

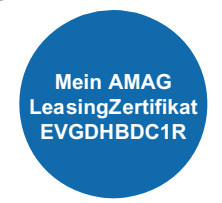

berechnet seinen monatlichen Maximalbetrag für ein Leasing

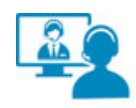

lässt sich anschliessend online identifizieren

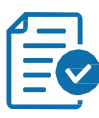

bekommt die verbindliche Zusage für einen Leasingvertrag

D Abb. 4.4 Zielsetzung: Online-Bewilligung

- Langfristig (Folgephase): Der Kunde erhält nicht nur eine Zusage für einen Leasingvertrag, sondern kann online ein Fahrzeug auswählen, einen Leasingvertrag abschliessen und einen Termin für die Fahrzeugübergabe beim Händler vereinbaren.

- Kundennutzen: Kunden, die ein Online-Leasing dem Besuch beim Händler vorziehen, können den kompletten Prozess online durchlaufen.

Mit dem Projekt setzt AMAG Leasing klare Signale für die digitale Ausrichtung der AMAG. Das Projekt Video-Identifikation kann wertvolle Erkenntnisse und Grundlagen für die Digitalisierung weiterer Prozesse und Geschäftsbereiche schaffen. Wesentlich für den Erfolg wird sein, ob es AMAG Leasing gelingt, die Kundenbedürfnisse bei der Gestaltung des Online-Leasings einzubeziehen und so auch neue Zielgruppen durch positive Kundenerlebnisse zu überzeugen.

॥ Wir stellen die Wirkung haptischer Erlebnisse und persönlicher Beziehungen im Automobilvertrieb nicht in Frage, aber es gibt Zielgruppen und Situationen, für die wir frühzeitig digitale Ergänzungen und Alternativen anbieten wollen. In der diskreten Online-Erfassung persönlicher Daten für einen Leasingantrag, von zuhause und jederzeit, komplettiert durch die unkomplizierte videobasierte Online-Identifikation, sehen wir einen echten Kundenutzen (Patrick Frauchiger, Leiter Verkauf und Marketing, AMAG Leasing AG).

\section{Digitalisierung in der Automobilbranche - Was will der Kunde?}

Nicht nur die Angebotsseite verändert sich, auch das Informations- und Entscheidungsverhalten der Automobilkunden ist im Wandel. Studien zeigen, dass Online-Kanäle nicht nur an Bedeutung gewinnen, sondern in der Phase der Informationsbeschaffung bereits dominieren. Aber auch Kundenerlebnisse im stationären Handel bleiben relevant:

- Mehr als $80 \%$ der Neuwagenkunden und nahezu $100 \%$ der Gebrauchtwagenkunden starten die Informationssuche online. Die Anzahl der Besuche beim Händler vor dem Autokauf sind von durchschnittlich 5 auf bis zu einem Besuch gesunken (abhängig von Region und Marke). Mehr als $80 \%$ der Kunden wollen jedoch auf die Probefahrt beim Händler nicht verzichten (Global Consumer Survey in USA, Europa und China (McKinsey \& Co. 2014)).

I) Der Händler verliert in der Anfangsphase die Informationshoheit über das Produkt (Detlev Mohr, Leiter Automotive Practice Europe, (McKinsey \& Co. 2014)). 
- Auch bei Fahrzeugleasing und -finanzierung ist das Internet zur wichtigsten Informationsquelle für Kunden geworden, wie eine Studie bei Endkunden in Deutschland zeigt (Ernest \& Young GmbH 2013). Mehr als $40 \%$ der befragten potenziellen Kunden, die über den Kauf oder die Finanzierung eines Fahrzeuges nachdenken oder in den letzten 12 Monaten ein Auto gekauft oder finanziert haben, nutzen das Internet, um sich über das Thema Leasing/Finanzierung zu informieren. Rund $24 \%$ informieren sich telefonisch oder persönlich beim Händler.

- Die Fahrzeugfinanzierung wird dabei für Konsumenten im Entscheidungsprozess zunehmend wichtiger. Auf die Frage, zu welchem Zeitpunkt sie sich über die Finanzierung ihres Neu- oder Gebrauchtwagens informieren, gaben 48,2\% der Befragten an, dass sie dies vor der Modellwahl, 29,4\% während und 14,2\% nach der Wahl des Modells tun (Ernest \& Young GmbH 2013).

- Die Studie empfiehlt Autobanken und Autohändlern auf diese Herausforderungen und Chancen gemeinsam mit Multikanalvertriebsstrategien zu reagieren. Weitere Potenziale werden in der Nutzung des Internets, über die statische Information hinaus, als Transaktionsplattform für das Leasing gesehen. Auch die Erweiterung des Angebots um neue Mobilitätskonzepte (z. B. Übernahme von Carsharing-Lizenzen, Bring-/Hohldienste) werden als gemeinsames Aktionsfeld für Handel und Leasinggeber vorgeschlagen (Ernest \& Young $\mathrm{GmbH}$ 2013).

\subsection{Umsetzung und Wirkung}

AMAG Leasing ist sich bewusst, dass sie mit der Online-Videoidentifikation Neuland im Schweizer Finanzdienstleistungsmarkt betritt. Die Möglichkeiten, die sich dadurch für die weitere Ausgestaltung des Online-Leasings bis hin zum digitalisierten Absatz ergeben, sollen daher schrittweise getestet und umgesetzt werden. Die Kundenbedürfnisse werden auf diesem Weg frühzeitig erhoben und in die Ausgestaltung einbezogen.

Um die oben beschriebenen Ziele zu erreichen, gliedert AMAG Leasing das Projekt in drei Phasen (• Abb. 4.5).
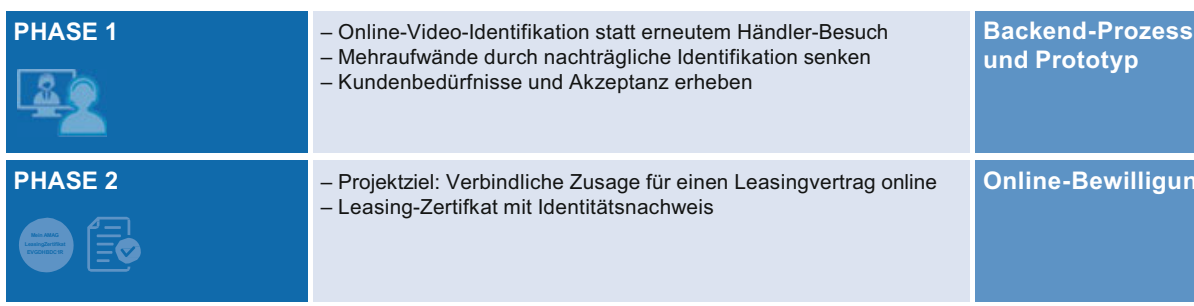

Online-Bewilligung

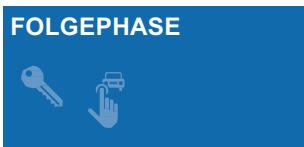

- Potenzial: Leasing und Fahrzeugauswahl online

- Abb. 4.5 Vorgehen und Inhalte der Phasen 


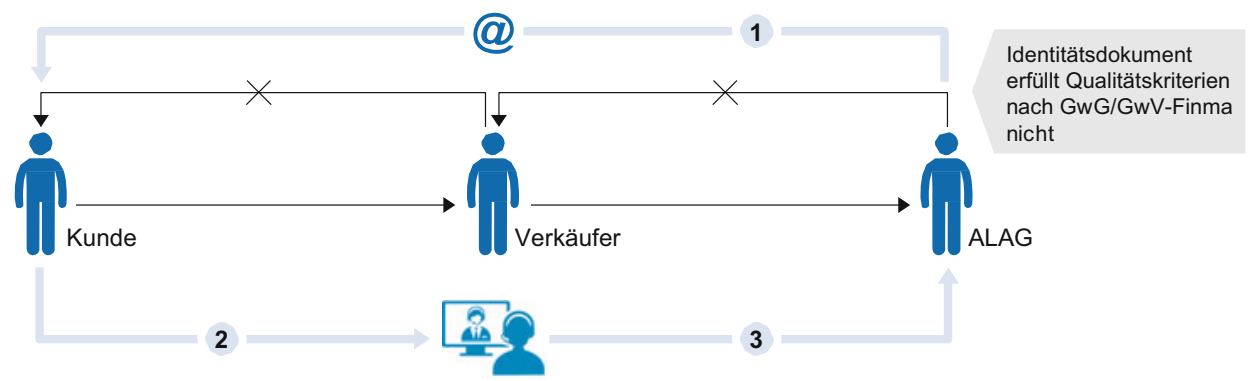

- Abb. 4.6 Phase 1-Backend-Prozess und Prototyp

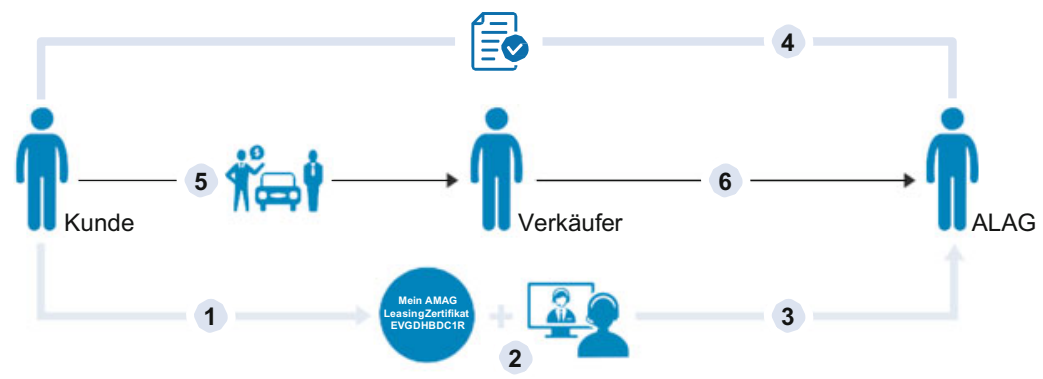

- Abb. 4.7 Phase 2-Online-Bewilligung

In der ersten Phase wird die Online-Videoidentifikation im bestehenden Prozess eingesetzt, um die Mehraufwände zu reduzieren, die entstehen, wenn der GwG-Check der Vertragsadministration Mängel beim Identitätsnachweis feststellt (- Abb. 4.6). AMAG Leasing muss in diesem Fall nicht mehr wie bis anhin den Händler auffordern, den Kunden erneut aufzubieten, um sich persönlich auszuweisen. Stattdessen wird aus dem Prozessmanagementsystem eine EMail an den Kunden mit einem Link zur Online-Videoidentifikation erstellt (1). Der Kunde identifiziert sich online (2) und die Daten werden an AMAG Leasing übermittelt (3). In dieser Pilotphase nutzt AMAG Leasing für die Online-Videoidentifikation die Dienstleistung eines Drittanbieters.

Nach erfolgreichem «Prototyping» wird die Zielgruppe der Online-Identifikation erweitert und das bereits bestehende Online-Leasingzertifikat in den Prozess integriert. In Phase 2 («Online-Bewilligung») sind nicht mehr nur Kunden, die sich erneut identifizieren, angesprochen, sondern alle potenziellen Kunden, die online einen Leasing-Antrag stellen möchten (- Abb. 4.7). Für die Eingabe ihrer persönlichen Daten nutzen Kunden das Online-Leasingzertifikat (1), das den finanziellen Rahmen, innerhalb dessen ein Leasing möglich ist, online prüft und bescheinigt. In einem nächsten Schritt werden im Video-Chat mit Web-ID die eingegebenen persönlichen Daten und die Identität der Person und deren Ausweis geprüft (siehe Infobox «FINMA ermöglicht Online-Videoidentifikation») (2). Die Daten werden an AMAG Leasing übermittelt (3), die dem Kunden auf dieser Grundlage online eine verbindliche Zusage für einen Leasingvertrag erteilen kann (4). Mit dieser Zusage kann der Kunde zum Händler, um ein Auto auszuwählen und den Vertrag zu unterzeichnen (5). Abschliessend wird die Vertragsadministration ausgelöst.

Mit der Online-Bewilligung ist das Projektziel für AMAG Leasing erreicht. In einer Folgephase werden anschliessend die Potenziale für den gesamten Absatzprozess betrachtet. In die- 


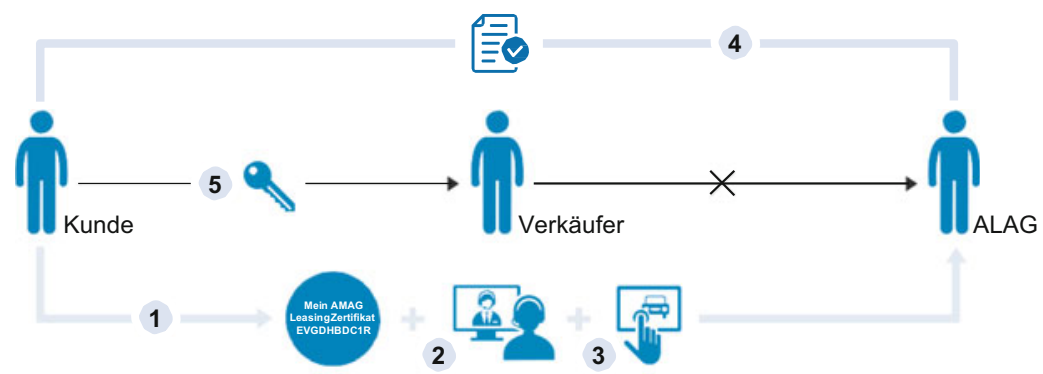

- Abb. 4.8 Folgephase-Online-Absatz

sem Szenario (- Abb. 4.8) erstellt sich der Kunde online ein Leasingzertifikat (1), lässt sich per Video-Chat identifizieren (2) und wählt ebenfalls online das Fahrzeug aus (3). Eine Schnittstelle zur webbasierten Fahrzeugauswahl, die Gegenstand eines laufenden Projekts ist, soll diesen Schritt ermöglichen. Die bestehende durchgängige Automatisierung würde zudem ermöglichen, dass nicht nur eine verbindliche Zusage für einen Leasingvertrag ausgestellt werden kann, sondern auch der Leasingvertrag online abgeschlossen werden kann (4). Abschliessend könnte der Kunde einen Termin für die Fahrzeugübergabe beim Händler (5) online vereinbaren. Die Frage der Verfügbarkeit des Fahrzeuges wird durch den hohen Standardisierungsgrad der Fahrzeuge in Bezug auf Motorisierung und Ausstattung zunehmend unwesentlich.

\section{- Die Lösungsarchitektur}

Für die Umsetzung der Szenarien hat die AMAG Leasing durch die Automatisierung des Bewilligungsprozesses systemseitig bereits wesentliche Voraussetzungen geschaffen. Das CRMSystem und das Vertragsmanagement-System sind in den automatisierten Prozess integriert. Auch die Middleware für den Datenaustausch ist bereits vorhanden. Für die Umsetzung der oben beschriebenen Phasen müssen lediglich die bestehenden Online-Instrumente LeasingZertifikat und Leasing-Rechner sowie der externe Videoidentifizierungsservice eingebunden werden (- Abb. 4.9).

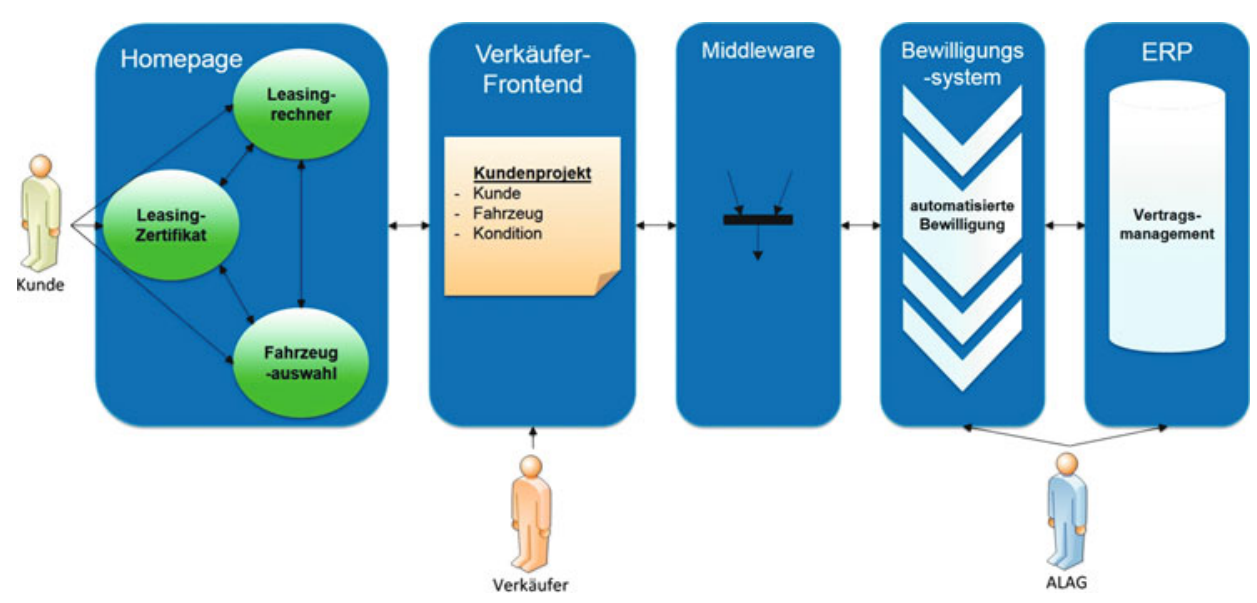

- Abb. 4.9 Systemlandschaft - Status quo und Erweiterungen für das Online-Leasing 


\section{- Was will der Kunde?}

Die grösste Herausforderung für AMAG Leasing ist nicht die systemseitige Implementierung des online erweiterten Leasingprozesses. Da sie mit der erst kürzlich in der Schweiz möglichen Video-Identifizierung Neuland beschreitet, ist die Akzeptanz der Schweizer Kunden nicht vorhersehbar. Auch die Bereitschaft für ein vollständig online durchgeführtes Fahrzeugleasing lässt sich kaum abschätzen.

Sind Schweizer Kunden bereit, vertrauliche Daten im Rahmen eines Video-Chats, in dessen Verlauf Fotos von ihnen und ihren Ausweisdokumenten erstellt werden, mit einer Drittpartei zu teilen? Sehen sie einen Vorteil darin, Daten zu ihrer persönlichen und finanziellen Situation online zu erfassen, um direkt eine verbindliche Zusage für ein Leasing zu erhalten? Und zu welchem Zeitpunkt befassen sie sich mit dem Thema Leasing? Bevor oder nachdem sie ihr Traumauto ausgewählt haben?

॥ Die Online-Bewilligung kann ein Alleinstellungsmerkmal für die AMAG Leasing werden, aber die Akzeptanz der videobasierten Identifikation durch Schweizer Kunden und deren Bedürfnisse beim Online-Leasing sind ungewiss. Vielleicht sind wir etwas früh für den Schweizer Markt? Aus diesem Grund testen wir verschiedene Szenarien mit extern rekrutierten Probanden (Esad Ceranic, Projektverantwortlicher, AMAG Leasing AG).

Um diese Fragen bereits vor der definitiven Implementierung des Online-Leasingprozesses zu klären, hat sich AMAG Leasing entschieden, mit dem Service Lab der Zürcher Hochschule für Angewandte Wissenschaften zusammenzuarbeiten. Mit Testkunden, die bereits Erfahrungen mit Fahrzeugleasing haben oder ein solches in Betracht ziehen, werden Interviews durchgeführt und die aktuellen Online-Tools sowie die Video-Identifikation über einen externen Service getestet. Als Ausgangspunkt für diesen qualitativen Ansatz wurden mit AMAG Leasing drei Szenarien erarbeitet, die unterschiedliche Zugänge zum Online-Leasing darstellen. Die Ergebnisse der Interviews und Beobachtungen werden eingesetzt, um den Zugang zum Online-Leasing möglichst einfach und bedürfnisgerecht zu gestalten. - Abb. 4.10 fasst Ziele und Vorgehen zusammen.

\section{- Erste Erkenntnisse}

Die Beobachtungen und qualitativen Tiefeninterviews mit den Probanden liefern für AMAG aufschlussreiche Erkenntnisse sowohl über die grundlegende Einstellung und den Wissensstand potenzieller Kunden zum Thema Leasing, als auch über deren Umgang mit den bestehenden Online-Tools und mit der Online-Identifikation.

Bezüglich der Bereitschaft, sich online identifizieren zu lassen, können die Befragten relativ klar in zwei Gruppen eingeteilt werden: während es für die einen nicht bzw. noch nicht in Frage kommt, ihr Gesicht und ihre ID im Internet in eine Kamera zu halten, sehen andere - vor allem unter dem Gesichtspunkt, dass es sich um eine moderne und zeitsparende Methode handelt darin kein Problem. Abgesehen von diesen Grundeinstellungen der Probanden hängt die Bereitschaft für eine solche Online-Identifikation auch vom Wissen der Befragten über Leasing ab. Haben diese in der Vergangenheit bereits einen Leasing-Vertrag abgeschlossen und wissen, dass im Prozess zu irgendeinem Zeitpunkt eine Identifikation auf sie zukommt, rechnen sie im Online-Prozess auch eher mit diesem Schritt. Personen, welche noch keine Erfahrung mit Leasing haben, reagieren entsprechend unvorbereitet und zurückhaltend auf die Situation. Unabhängig von Wissen und Einstellungen ist die Identifizierung über das Internet für alle Probanden ein neuer Anwendungsfall, der Verunsicherung auslöst. Eine wichtige Rolle spielt 


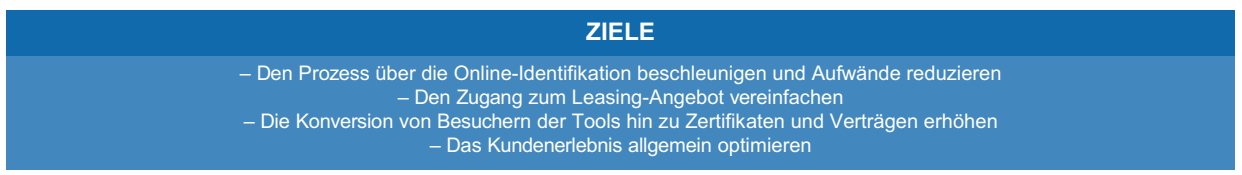

SZENARIO 1: BUDGET

Der Kunde hat ein Budget, das einzuhalten ist. Mit diesem im Hinterkopf macht er sich an den Online-Leasing-Prozess.

SZENARIO 2: AUTO

Der Kunde hat online oder beim Händler sein Traumauto gesehen. Dies möchte er nun online leasen.

SZENARIO 3: ONLINE-SHOPPING

Aufgrund des Leasingzertifikats, das der Kunde erhalten hat, werden inm zum Budget passende Autos vorgeschlagen.

\begin{tabular}{|c|c|c|}
\hline$\downarrow$ & $\downarrow$ & $\downarrow$ \\
\hline EINSTELLUNG/ERWARTUNG & AKTUELLEONLINE-TOOLS & $\begin{array}{l}\text { VIDEOBASIERTE } \\
\text { ONLINE- } \\
\text { IDENTIFIKATION }\end{array}$ \\
\hline $\begin{array}{l}\text { Zugang Leasing-Prozess ALAG } \\
\text { - Warm-Up } \\
\text { - Erfahrungen mit Leasing } \\
\text { - Spontanes Vorgehen bei einem } \\
\text { solchen Prozess } \\
\text { - Erwartungen an Onlineprozess }\end{array}$ & $\begin{array}{l}\text { Leasingrechner und Zertifikat } \\
\text { - Spontane Reaktionen } \\
\text { - Ausführen der Tools mit Thinking-aloud } \\
\text { - Likes und Dislikes } \\
\text { - Treiber und Hürden }\end{array}$ & $\begin{array}{l}\text { Prozess durchgehen } \\
\text { - Spontane Reaktionen } \\
\text { - Prozessdurchführung } \\
\text { - Likes und Dislikes } \\
\text { - Treiber und Hürden } \\
\text { - Akzeptanz }\end{array}$ \\
\hline
\end{tabular}

ERGEBNISSE

- Mindset bezüglich Leasingprozess

- Bedürfnis- und Motivstrukturen

- Im Vorfeld wahrgenommene «gains» und "pains»

- Einstellungen

- Verständlichkeit und «Handhabung» der Tools
- Handlungsempfehlungen zur Verbesserung der aktuellen Tools

- Wie gehen die Befragten mit dem Web-ID Prozess um

(inkl. Akzeptanz)

- Abschliessende Gesamtbeurteilung

- Abb. 4.10 Kooperation mit dem ZHAW Service Lab

dabei die Rahmenbedingung, dass die Online-Identifikation über einen Drittanbieter durchgeführt wird: auch wenn die Online-Identifikation in die amag.ch-Seite eingebettet ist und die Nutzer vor Aufbau der Livesitzung darauf hingewiesen werden, dass sie mit einem externen Partner für die Online-Identifikation verbunden werden, ist das Vertrauen in einen Drittanbieter nicht in gleichem Umfang gegeben wie in die bekannte AMAG. Die Interviewten fragen sich unter anderem, wie das Unternehmen die Daten handhabt und wie die Datensicherheit gewährleistet wird. Diese nicht zwingend rational begründeten Zweifel haben zur Folge, dass einige der Probanden den Gang zum Händler bevorzugen würden, um sich identifizieren zu lassen. Eine weitere Erkenntnis aus den Interviews und Beobachtungen ist, dass die Bereitschaft zu einer Online-Identifikation und zu einem Online-Leasing-Prozess insgesamt steigt, wenn die Interviewten mit der Auswahl des Autos in den Prozess einsteigen.

Auf der Grundlage dieser und weiterer Erkenntnisse wurden Handlungsempfehlungen formuliert, die kurzfristig umsetzbare operative Massnahmen und mittel- bis langfristige strategische Massnahmen umfassen. Zentral wiegt dabei die Erkenntnis, dass, unabhängig vom untersuchten Szenario, ein Grundbedürfnis nach Transparenz und Unterstützung im gesamten Prozess auf Kundenseite besteht. Dabei geht es nicht nur um grundlegende Informationen zum Thema Leasing und eine Erklärung der Schritte im Leasingprozess und dessen Fortschritt, sondern um eine verständliche Aufklärung zu Beginn und prozessbegleitend, welche Informationen und Aktivitäten zum Abschluss der einzelnen Schritte notwendig sind.

- Ausblick

AMAG Leasing ist sich bewusst, dass für den Erfolg eines durchgängigen Online-Leasings nicht nur technische Lösungen und optimal gestaltete Oberflächen entscheidend sind. Die beschrie- 
benen Neuerungen im Leasingprozess wirken sich massiv auf interne Abläufe, Strukturen und Rollenverteilungen aus, die über das Leasing hinausgehen und den gesamten Fahrzeugabsatz betreffen. Die Veränderungen in der Kundenbeziehung und im Kundenverhalten müssen von allen beteiligten Akteuren entlang der Absatzkette getragen werden. AMAG Leasing hat sich entschieden, diese zentralen Fragestellungen auf der Grundlage erster Erfahrungen schrittweise zu adressieren. Abzuwarten, bis alle organisatorischen, betriebspolitischen und rechtlichen Fragestellungen abschliessend geklärt sind, lässt der Wettbewerbsdruck in einer zunehmend digitalisierten Automobilbranche nicht zu. Klar an den Ausgangspunkt gestellt werden hingegen die Bedürfnisse, Reaktionen und Einstellungen der Kunden. Zum Zeitpunkt der Erstellung der vorliegenden Fallstudie befindet sich AMAG Leasing in der Phase des «Prototyping» der Online-Videoidentifikation. Parallel werden die Erkenntnisse aus den Interviews und Beobachtungen mit Probanden im Service Lab ausgewertet und die operativen und strategischen Handlungsempfehlungen für die Optimierung der Online-Angebote sowie die Ausgestaltung der Digitalisierungsstrategie eingesetzt.

\subsection{Fazit}

Die vorliegende Fallstudie von AMAG Leasing zeigt, wie eine im Back-End begonnene digitale Transformation für die Schaffung neuer Kundenerlebnisse genutzt wird. Ausgehend von einem durchgängig automatisierten Bewilligungsprozess kann die Tür zum Kunden geöffnet und ein Online-Leasing angeboten werden. Machbar wird dies durch veränderte regulatorische Rahmenbedingungen, die eine Online-Videoidentifikation für Finanzdienstleistungskunden in der Schweiz neu zulassen. Auf dieser Grundlage kann AMAG Leasing Geschäftsbeziehungen mit Kunden GwG-konform online eröffnen und ihnen eine Alternative zum Weg über den stationären Handel bieten.

Die Fallstudie deckt ein breites Spektrum der im Rahmen der Studie erforschten Aspekte der digitalen Transformation ab. Das Vorhaben verändert die Domänen Kunde, Geschäftsmodell und Business Operations gleichermassen und macht die Relevanz der Prozessdigitalisierung deutlich. Die wesentlichen Erkenntnisse sind in - Abb. 4.11 gekennzeichnet und nachfolgend dargestellt.

Mit welcher Zielsetzung und mit welcher Wirkung wird digital transformiert? Welcher Kundennutzen wird angestrebt bzw. wurde bereits realisiert?

1 Kundennutzen: Online-Alternative zum Händlerbesuch

In einem ersten Schritt profitieren bestehende Kunden, deren Fahrzeug bereits ausgeliefert ist und deren Ausweisdokument die GwG-Qualitätsprüfung nicht besteht. Sie ersparen sich den Weg zum Händler und können sich bequem online im Video-Chat erneut identifizieren. Mit der Integration des Online-LeasingRechners und des Online-Leasingzertifikats werden Zielgruppe und Nutzen erweitert. Der komplette Leasingantrag kann diskret und bequem online gestellt werden und führt bei positiver Bewilligung direkt zur verbindlichen Zusage. Wird die Auswahl des Fahrzeuges zukünftig integriert, können Kunden, die diese Form der Interaktion einem Besuch beim Händler vorziehen, den kompletten Prozess online durchlaufen. Um sicherzustellen, dass das Online-Leasing tatsächlich positive Kundenerlebnisse schafft und neue Zielgruppen überzeugt, werden Kundenbedürfnisse frühzeitig in die Ausgestaltung einbezogen. 


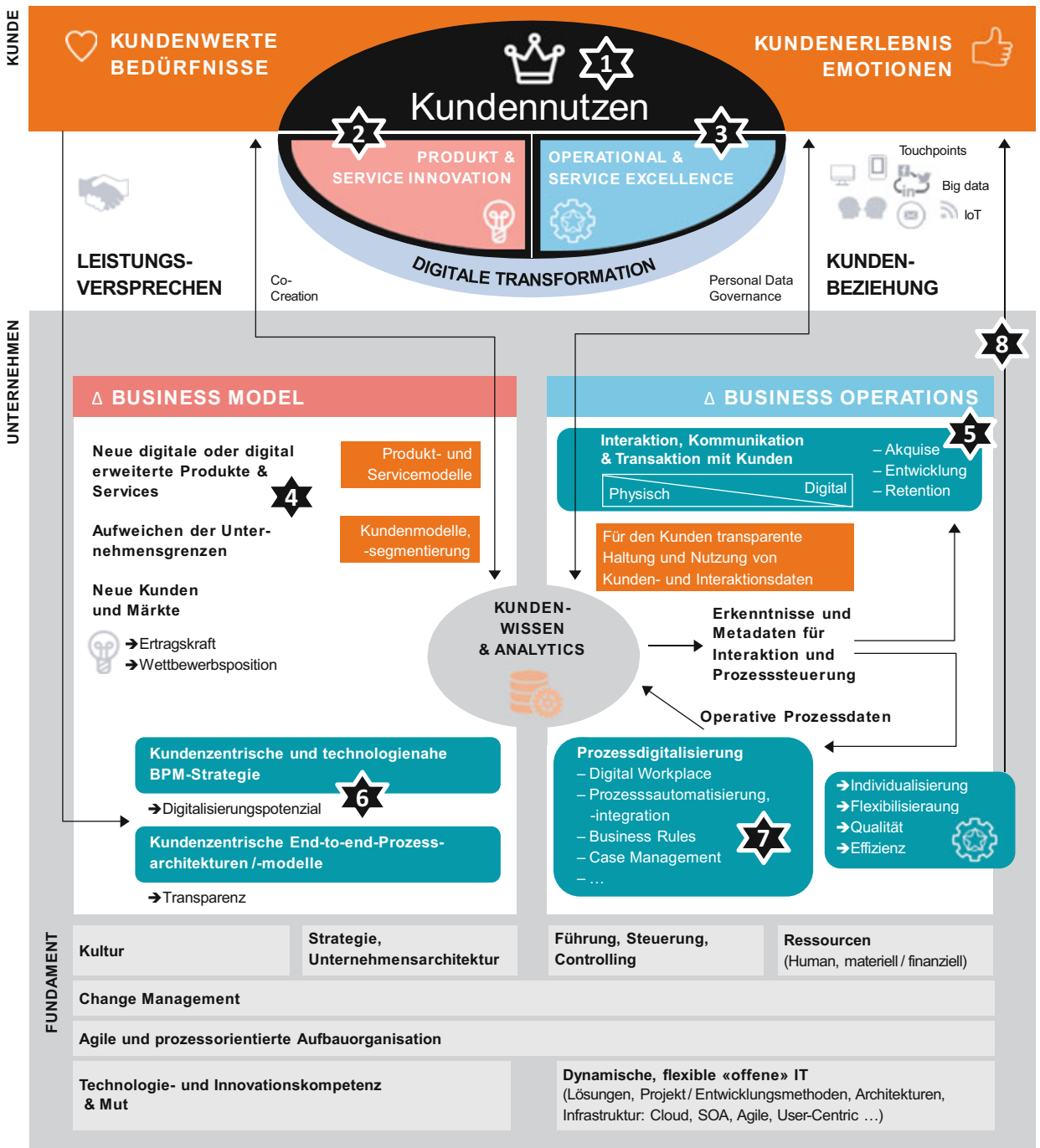

- Abb. 4.11 AMAG Leasing Fallstudie - Kernaspekte im Kontext des Studienframeworks

\section{Produkt- und Service-Innovation}

Das Serviceangebot der AMAG wird durch das Online-Leasing digital erweitert. Ein durchgängiges OnlineLeasing ohne Medienbrüche war in der Schweiz aufgrund der fehlenden Rechtsgrundlagen für die OnlineIdentifikation bisher nicht möglich. AMAG Leasing kann diese Lücke schliessen und Wettbewerbsvorteile sichern. Der veränderte Prozess bietet Anknüpfungspunkte für weitere Innovationen; so könnten Carsharing-Modelle in das Online-Leasing eingebunden und angeboten werden. Aktuell lassen viele Leasinganbieter Carsharing nicht zu. Die AMAG Gruppe hat mit ihrer Beteiligung am Zürcher Startup Sharoo und Catch a Car von Mobility signalisiert, dass sie offen ist, diese Formen der Mobilität in ihr Geschäftsmodell zu integrieren. 


\section{Operational \& Service Excellence}

Ein Auslöser für das Vorhaben waren die Mehraufwände, die durch mangelhafte Identitätsnachweise in der Vertragsadministration und in der Folge beim Händler und letztlich beim Kunden entstehen. Wird die Online-Videoidentifikation von den Kunden akzeptiert, können die Prozesskosten reduziert und die Kundenzufriedenheit erhöht werden. Auch das Online-Leasing mit Neukunden profitiert von dieser Durchgängigkeit des Leasingprozesses von der Eröffnung der Geschäftsbeziehung bis hin zur Vertragsadministration.

\section{Was wurde bzw. wird digital transformiert?}

\section{Business Model: Neue Rolle für AMAG Leasing}

Traditionell wird ein Fahrzeuggeschäft, sei es Kauf oder Leasing, über den stationären Handel angebahnt. Die Möglichkeit einen Leasingantrag rechtskonform vollständig online abzuwickeln, bietet der AMAG Leasing neue Möglichkeiten in der Interaktion mit dem Kunden und in der Unterstützung des Händlers in der Geschäftsanbahnung insbesondere mit Blick auf die stetig zunehmenden gesetzlichen Regulierungen. Der stationäre Handel bleibt für das Kundenerlebnis jedoch nicht nur bei Probefahrten oder beim Service relevant und es gilt daher, die optimale Rollenverteilung im veränderten Geschäftsmodell zu finden. Eine weitere Veränderung erfährt das Geschäftsmodell, indem das Servicesystem für das Online-Leasing um ein Drittpartei erweitert wird, die durch die Online-Identifizierung ein durchgängiges Kundenerlebnis ermöglicht.

\section{Business Operations: Digital und physisch}

Mit dem automatisierten Bewilligungsprozess ist bei AMAG Leasing bereits ein stabiles operatives Rückgrat für das Online-Leasing vorhanden. Effizienz und Produktivität des Leasingprozesses wurde auf dieser Grundlage deutlich gesteigert und fortlaufend verbessert. Mit der Online-Videoidentifikation und dem Online-Leasing wird die Reichweite dieses optimierten Prozesses nun in Richtung Kunde erweitert. Dabei gilt es digitale und physische Kundenerlebnisse durchgängig zu gestalten. Beim automatisierten Bewilligungsprozess ist die optimale Einbindung des Handels bereits gelungen und auch die Erfahrungen mit dem Online-Leasingzertifikat zeigen, dass Kunden einen online begonnenen Prozess im stationären Handel vollenden können.

\section{$\widehat{6}$ End-to-End-Prozessarchitektur und technologienahe BPM-Strategie}

Die End-to-End-Prozesse im Automobilvertrieb betreffen alle AMAG Gesellschaften. Die Entwicklung der Prozessarchitektur und die fachliche Prozessmodellierung findet daher auf Ebene AMAG-Gruppe statt. AMAG Leasing nutzt diese für die Prozessoptimierung. Auf Impulse in Richtung Digitalisierungspotenzial wartet AMAG Leasing jedoch nicht, sondern setzt hier auf die Erkenntnisse aus den umgesetzten Automatisierungsprojekten.

\section{Wie und wodurch wird transformiert? \\ 7 Prozessdigitalisierung: Automatisierung und Integration im Back-End}

Kunden ein Online-Leasing anzubieten, das innert Minuten, das heisst in nahezu Echtzeit, nicht nur Informationen zum finanzierbaren Rahmen liefert, sondern auch eine verbindliche Zusage erteilt, bedingt ein hohes Mass an Systemintegration und implementierter Geschäftslogik im Back-End. Mehr als 200 Geschäftsregeln waren im Rahmen des automatisierten Bewilligungsprozesses bereits implementiert und weiterentwickelt worden. Alle relevanten internen und externen Systeme waren bereits integriert. 


\section{Prozessdigitalisierung: Orchestrierung im Front-End}

Die Integration der bestehenden Tools für das Online-Leasing in diese Infrastruktur stellt technisch keine grosse Herausforderung für AMAG Leasing dar. Der Fokus liegt vielmehr auf der optimalen Ausgestaltung des Prozesses aus der Perspektive des Kunden. Kunden haben unterschiedliche Bedürfnisse und Ausgangssituationen für das Online-Leasing (z. B. Fahrzeugwunsch, fixes Budget). AMAG Leasing prüft, ob der digitalisierte Prozess verschiedene Einstiegspunkte bieten und sich idealerweise dem Kundenverhalten anpassen muss, um ein individuelles Kundenerlebnis auch in der Online-Welt zu bieten. Ein noch nicht vollständig ausgeschöpftes Potenzial stellen in diesem Zusammenhang die operativen Daten dar, die AMAG Leasing durch die Prozessautomatisierung bereits gewonnen hat. Auf dieser Grundlage wären sowohl retrospektiv als auch prädiktiv Rückschlüsse auf das Kundenverhalten möglich.

\section{Literatur}

Adam, S., \& Meyer, M. (2015). Integration der Kundenperspektive als Basis für Bedarfsorientierung und Weiterentwicklung integrierter Mobilitätsplattformen. In Marktplätze im Umbruch (S. 589-601). Berlin Heidelberg: Springer.

Berger, H., \& Rechenbach, M. (2015). Financial Services follows automotive? - Digitalisierung des Automobilvertriebs und Konsequenzen für die automobile Finanzdienstleistung. In Handbuch Automobilbanken (S. 167-181). Berlin Heidelberg: Springer.

Ernest \& Young GmbH (2013). Multikanalvertrieb - Erfolgsschlüssel für Automobilhandel und Autobanken: Executive Summary der EY-Studie 2013. http://www.ey.com/Publication/vwLUAssets/EY_Erfolgsschluessel_ fuer_Automobilhandel_und_Autobanken/\$FILE/EY-Chancen-fuer-Autobanken.pdf. Abgerufen am 5.6.2016.

FINMA (2016a). FINMA-Medienmitteilung: »FINMA baut Hürden für Fintech ab«. https://www.finma.ch/de/news/ 2016/03/20160317-mm-fintech/ (Erstellt: 17. März 2016). Abgerufen am 5.6.2016.

FINMA (2016b). FINMA-RS 16/7 »Video- und Online-Identifizierung". Sorgfaltspflichten bei der Aufnahme von Geschäftsbeziehungen über Kanäle: Erlass. Inkraftsetzung: 18. März 2016.

GwG. Bundesgesetz über die Bekämpfung der Geldwäscherei und der Terrorismusfinanzierung (Geldwäschereigesetzt (GwG) vom 10. Oktober 1997) (Stand 1. Januar 2016).

GwV-FINMA. Verordnung vom 3. Juni 2015 der Eidgenössischen Finanzmarktaufsicht über die Bekämpfung von Geldwäscherei und Terrorismusfinanzierung im Finanzsektor (Geldwäschereiverordnung-FINMA, GwV-FINMA), SR 955.033.0 (Stand 1. Januar 2016).

McKinsey \& Co (2014). Innovating automotive retail Journey towards a customer-centric, multiformat sales and service network. https://www.mckinsey.de/innovating-automotive-retail. Abgerufen am 5.6.2016.

Singh, S. (2014). The future of car retailing. Forbes online. http://www.forbes.com/sites/sarwantsingh/2014/02/05/ the-future-of-car-retailing/\#245e572e2d51. Abgerufen am 5.6.2016.

Wedeniwski, S. (2015). Mobilitätsrevolution in der Automobilindustrie: Letzte Ausfahrt digital! Berlin Heidelberg: Springer.

Open Access Dieses Kapitel wird unter der Creative Commons Namensnennung 4.0 International Lizenz (http://creativecommons.org/licenses/by/4.0/deed.de) veröffentlicht, welche die Nutzung, Vervielfältigung, Bearbeitung, Verbreitung und Wiedergabe in jeglichem Medium und Format erlaubt, sofern Sie den/die ursprünglichen Autor(en) und die Quelle ordnungsgemäß nennen, einen Link zur Creative Commons Lizenz beifügen und angeben, ob Änderungen vorgenommen wurden.

Die in diesem Kapitel enthaltenen Bilder und sonstiges Drittmaterial unterliegen ebenfalls der genannten Creative Commons Lizenz, sofern sich aus der Abbildungslegende nichts anderes ergibt. Sofern das betreffende Material nicht unter der genannten Creative Commons Lizenz steht und die betreffende Handlung nicht nach gesetzlichen Vorschriften erlaubt ist, ist für die oben aufgeführten Weiterverwendungen des Materials die Einwilligung des jeweiligen Rechteinhabers einzuholen. 